\title{
Fast-ion velocity-space tomography using slowing-down regularization in EAST plasmas with co- and counter-current neutral beam injection Paper
}

Madsen, B.; Huang, J.; Salewski, Mirko; Järleblad, H.; Hansen, P.C.; Stagner, L.; Su, J.; Chang, J. F.; Fu, J.; Wang, J. F.

Total number of authors:

24

Published in:

Plasma Physics and Controlled Fusion

Link to article, DOI:

10.1088/1361-6587/abb79b

Publication date:

2020

Document Version

Peer reviewed version

Link back to DTU Orbit

Citation (APA):

Madsen, B., Huang, J., Salewski, M., Järleblad, H., Hansen, P. C., Stagner, L., Su, J., Chang, J. F., Fu, J., Wang, J. F., Liang, L. Z., Zhong, G. Q., Li, Y. Y., Lyu, B., Liu, H. Q., Zang, Q., Luo, Z.' P., Nocente, M., Moseev, D., ... Liao, L. Y. (2020). Fast-ion velocity-space tomography using slowing-down regularization in EAST plasmas with co- and counter-current neutral beam injection: Paper. Plasma Physics and Controlled Fusion, 62(11), [115019]. https://doi.org/10.1088/1361-6587/abb79b

\section{General rights}

Copyright and moral rights for the publications made accessible in the public portal are retained by the authors and/or other copyright owners and it is a condition of accessing publications that users recognise and abide by the legal requirements associated with these rights.

- Users may download and print one copy of any publication from the public portal for the purpose of private study or research.

- You may not further distribute the material or use it for any profit-making activity or commercial gain

- You may freely distribute the URL identifying the publication in the public portal 
ACCEPTED MANUSCRIPT

\section{Fast-ion velocity-space tomography using slowing-down regularization in EAST plasmas with co- and counter-current neutral beam injection}

To cite this article before publication: Birgitte Madsen et al 2020 Plasma Phys. Control. Fusion in press https://doi.org/10.1088/1361-6587/abb79b

\section{Manuscript version: Accepted Manuscript}

Accepted Manuscript is "the version of the article accepted for publication including all changes made as a result of the peer review process, and which may also include the addition to the article by IOP Publishing of a header, an article ID, a cover sheet and/or an 'Accepted

Manuscript' watermark, but excluding any other editing, typesetting or other changes made by IOP Publishing and/or its licensors"

This Accepted Manuscript is $\odot 2020$ The Author(s). Published by IOP Publishing Ltd..

During the embargo period (the 12 month period from the publication of the Version of Record of this article), the Accepted Manuscript is fully protected by copyright and cannot be reused or reposted elsewhere.

As the Version of Record of this article is going to be / has been published on a subscription basis, this Accepted Manuscript is available for reuse under a CC BY-NC-ND 3.0 licence after the 12 month embargo period.

After the embargo period, everyone is permitted to use copy and redistribute this article for non-commercial purposes only, provided that they adhere to all the terms of the licence https://creativecommons.org/licences/by-nc-nd/3.0

Although reasonable endeavours have been taken to obtain all necessary permissions from third parties to include their copyrighted content within this article, their full citation and copyright line may not be present in this Accepted Manuscript version. Before using any content from this article, please refer to the Version of Record on IOPscience once published for full citation and copyright details, as permissions will likely be required. All third party content is fully copyright protected, unless specifically stated otherwise in the figure caption in the Version of Record.

View the article online for updates and enhancements. 


\title{
Fast-ion velocity-space tomography using slowing-down regularization in EAST plasmas with co- and counter-current neutral beam injection
}

\author{
B. Madsen ${ }^{1}$, J. Huang ${ }^{2}$, M. Salewski ${ }^{1}$, H. Järleblad ${ }^{1}$, \\ P. C. Hansen ${ }^{4}$, L. Stagner ${ }^{3}$, J. Su ${ }^{2}$, J. F. Chang ${ }^{2}$, J. Fu ${ }^{2}$, \\ J. F. Wang ${ }^{2}$, L. Z. Liang ${ }^{2}$, G. Q. Zhong ${ }^{2}$, Y. Y. Li ${ }^{2}$, \\ B. Lyu $^{2}$, H. Q. Liu ${ }^{2}$, Q. Zang ${ }^{2}$, Z. P. Luo ${ }^{2}$, M. Nocente ${ }^{5}$, \\ D. Moseev ${ }^{6}$, T. S. Fan ${ }^{7}$, Y. M. Zhang ${ }^{7}$, D. Yang7 \\ J. Q. Sun ${ }^{7}$, L. Y. Liao ${ }^{7}$ and the EAST team \\ ${ }^{1}$ Department of Physics, Technical University of Denmark, Kgs. Lyngby, \\ Denmark \\ 2 Institute of Plasma Physics, Chinese Academy of Sciences, P.O. 1126, 230031 \\ Hefei, Anhui, China \\ 3 General Atomics, San Diego, CA, USA \\ ${ }^{4}$ Department of Applied Mathematics and Computer Science, Technical \\ University of Denmark, Kgs. Lyngby, Denmark \\ ${ }^{5}$ Department of Physics, University og Milano-Bicocca, Milano, Italy \\ ${ }^{6}$ Max-Planck-Institut für Plasmaphysik, Greifswald, Germany \\ ${ }^{7}$ School of Physics and State Key Laboratory of Nuclear Physics and \\ Technology, Peking University, Beijing, People's Republic of China \\ E-mail: birma@fysik.dtu.dk, juan.huang@ipp.ac.cn \\ September 2020
}

\section{Abstract.}

We demonstrate $2 \mathrm{D}$ reconstructions of the fast-ion velocity distribution from two-view fast-ion D-alpha (FIDA) measurements at the EAST tokamak. By expressing the distribution in a basis relying on the fast-ion slowing-down process in fusion plasmas, the smoothness and velocity-space resolution of reconstructions are improved. We reconstruct distributions of fast ions born from simultaneous co- and counter-current neutral beam injection and detect the expected distinct change in fast-ion birth pitch when comparing discharges utilizing different neutral beam injectors. For purely co-current injection, we find a good agreement between TRANSP-predicted and reconstructed fast-ion densities, pressures and current densities for energies above $20 \mathrm{keV}$. We furthermore illustrate the improvement of the reconstructed high-energy range $(>40 \mathrm{keV})$ of the distribution by combining FIDA with neutron emission spectroscopy (NES) measurements with the compact single-plate EJ301 scintillator.

Keywords: velocity-space tomography, fast-ion D-alpha spectroscopy, fast ions, slowing-down regularization, EAST tokamak

Submitted to: Plasma Phys. Control. Fusion 


\section{Introduction}

Fast ions play a key role in present-day tokamak operation. Loss of fast ions can lead to reduced heating efficiency and can damage plasma-facing components [1, 2]. In addition, fast ions will be vital for the next generation tokamaks such as ITER and CFETR which will strongly rely on continuous plasma heating through collisions with highly energetic fusion-born alpha particles $[3[6]$. Studies on the fast-ion distribution during various scenarios in different experimental devices are, therefore, of utmost importance. Through tomographic inversion, the fastion distribution can be reconstructed from combined measurements by multiple fast-ion diagnostics 7 9. Fast-ion velocity-space tomography has been especially well-established at the ASDEX Upgrade tokamak where the five-view fast-ion D-alpha (FIDA) diagnostic setup offers excellent coverage of velocity space allowing reliable reconstructions of the fast-ion distribution $10-13$. Previous studies have shown that reconstructing the velocity distribution function from a diagnostic system with incomplete coverage of velocity space is a challenging task 7, 11, 14 16. Specifically at the EAST tokamak, the two-view FIDA diagnostic 17, 18 is sensitive to fast ions in substantial regions of velocity space, but leave other regions covered by only one view [19. To nevertheless enable reliable reconstructions of the fast-ion distribution from sparse datasets, a palette of prior information has been developed [14, 15, 20]. We here introduce a new approach to include prior information in reconstruction, problems. This approach relies on changing the basis, in which the distribution is described, to one that carries a physical understanding of the behaviour of fast ions in fusion plasmas, namely the slowing down of ions through Coulomb collisions with the plasma constituents. Employing this, we reconstruct fast-ion distributions created from simultaneous coand counter-current injection from two neutral beam injectors (NBIs) in the EAST tokamak. This type of distribution has a more complex structure than singlebeam distributions which have until now been the main focus in tomographic studies on the fast-ion velocity distribution in NBI-heated plasmas.

The tomographic approach/requires knowledge of the velocity-space sensitivities of the employed diagnostics. This is encoded in weight functions that have been developed for collective Thomson scattering (CTS) 21], FIDA 22, 23], fast-ion loss detectors (FILD) 24], neutron emission spectroscopy (NES) 25, 26, and gamma-ray spectroscopy (GRS) [27, 28]. By combining measurements from different diagnostic methods in integrated fast-ion velocityspace tomography, the velocity-space resolution and coverage can be improved. This has been successfully pursued for combined FIDA and CTS measurements at ASDEX Upgrade [29], NES and GRS at JET 30, 31] and for synthetic CTS and GRS signals envisioned for ITER 16. At EAST, weight functions have recently been developed for three commissioned NES diagnostics $32-35$. Whereas FIDA is mostly sensitive to fast ions with energies near or below the neutral beam injection energy (typically $\sim 10-100 \mathrm{keV}$ ) due to the relative-speed dependence of the chargeexchange probabilities 36 , NES diagnostics generally have increased sensitivity above the injection energy as a result of the relative-energy dependence of the fusion cross-section. Combining FIDA with NES for fast-ion velocity-space tomography, therefore, offers simultaneous measurements of $\mathrm{keV}$ - and $\mathrm{MeV}$-range ions. In this work, we present the first reconstructions from combined FIDA and NES spectra by employing measurement from the compact single-plate EJ301 scintillator installed at EAST. The approach can be further extended to include also the compact stilbene scintillator and the time-of-flight enhanced diagnostics (TOFED) 35,37.

This paper is structured as follows. Section 2 provides an overview of the EAST setup and discharges studied in this work. The formalism of changing the basis of tomographic inversions is presented in section 3 and tested on synthetic signals in section 4 . In section 5, this approach is applied to measurements in a selection of EAST discharges heated by various NBI sources. The tomographic dataset is extended to also contain NES measurements in section 6. In section 7 . derived quantities such as the fast-ion pressure and current density are computed from measurement-based reconstructions. Section 8 concludes the paper.

\section{Experimental approach}

Four NBIs are installed at the EAST tokamak: two co-current injectors (NBI1R and NBR1L) from the Aport and two counter-current injectors (NBI2R and NBI2L) from the F-port, as illustrated in the top-view schematic of EAST in figure 1. NBI1L serves as the 
(a)
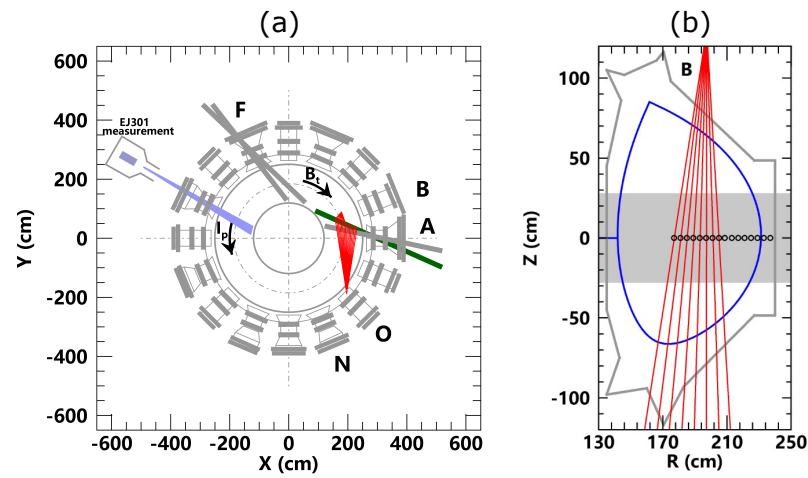

Figure 1. (a) Top-view and (b) poloidal cross-section of the EAST tokamak showing the four neutral beams and the locations of the O- and B-port FIDA views (red lines) as well as the EJ301 line-of-sight. The major and minor radii are $1.85 \mathrm{~m}$ and $0.45 \mathrm{~m}$, respectively.

diagnostic beam for the tangential O-port and vertical B-port FIDA views and is utilized in all discharges with FIDA measurements. The discharges studied in this work additionally employ one of the other beams. The full injection energies and powers of the NBI configurations employed here are given in table 1.

Discharges \#75439, \#75448 and \#75455 serve as reference shots used to evaluate the capabilities of purely FIDA-based reconstructions for various combinations of NBI injection in section 4 and section 5. These discharges were operated in Lmode and reached similar parameters. The electron temperature was $T_{e} \sim 0.2 \mathrm{keV}$, the line-averaged electron density was $n_{e} \sim 3 \times 10^{19} \mathrm{~m}^{-3}$ and the normalized beta value was $\beta_{N} \sim 1-1.1$. The discharges had a clockwise toroidal magnetic field of $B_{t} \sim 2.4 \mathrm{~T}$ on the magnetic axis and a counterclockwise plasma current of $I_{p} \sim 600 \mathrm{kA}$, as also indicated in figure 1. Due to higher neutron counts and hence improved measurement statistics for the EJ301 compact single-plate scintillator, discharge \#75469 offers the opportunity to combine FIDA and NES measurements in integrated data analysis in section 6 . Discharge \#75469 was operated in H-mode and was additionally heated by electron cyclotron resonance heating with a power of $0.55 \mathrm{MW}$ during measurement sampling. For this discharge, the electron temperature and normalized beta value reached $T_{e} \sim 0.3-1 \mathrm{keV}$ and $\beta_{N} \sim 1$, respectively.

During the discharges, the main heating beam (NBI1R, NBI2R or NBI2L) continuously injected particles, whereas the diagnostic beam (NBI1L) was modulated in order to allow FIDA background subtraction. This modulation is illustrated for discharge \#75469 in figure 2a. From the neutron counts simultaneously measured by the EJ301 scintillator in figure 2 p, it is

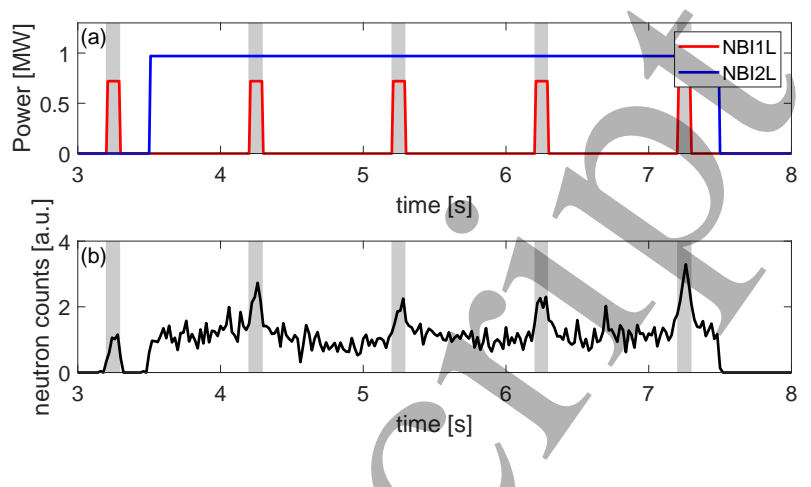

Figure 2. Time traces of the (a) NBI powers and (b) EJ301 neutron counts during discharge \#75469. The shaded areas enclose NBI1L blips.

evident that the NBI1L beam modulation strongly affects the neutron production. As the neutron production is dependent on the fast ions, we consider only the distribution during dual-beam injection

The neoclassically expected fast-ion velocity distributions close to the plasma center during dualbeam injections are given in figure 3. These are computed with the TRANSP/NUBEAM code 38. The distributions are expressed in the commonly used energy-pitch coordinates given as $E=\frac{1}{2} m_{i} v^{2}$ and $p=v_{\|} / v$. Here $m_{i}$ is the fast-ion mass, $v=$ $\sqrt{v_{1}^{2}+v_{\|}^{2}}$ is the fast-ion speed and the subscripts of the velocity components $v_{\perp}$ and $v_{\|}$indicate the directions relative to the magnetic field. Drifting Maxwellian distributions, as described in 39, with estimated local bulk ion temperatures, densities, and drift velocities: $T \sim 1.2-1.5 \mathrm{keV}, n \sim 2.5-2.8 \times 10^{19} \mathrm{~m}^{-3}$ and $v_{d} \sim 2.5-8 \mathrm{~km} / \mathrm{s}$, have been added to the TRANSP distributions in order to emphasize the much higher ion densities at energies below $10 \mathrm{keV}$ compared to the dilute populations at higher energies.

\subsection{FIDA and EJ301 measurements}

Using tomography, we search for the most likely local fast-ion distribution given the available measurements. From the FIDA diagnostics, we obtain information about the fast-ion velocity distribution through measurements of the Doppler-shifted Balmer-alpha radiation following charge exchange between injected neutrals and fast deuterium ions [40,41]. The EJ301 scintillator, on the other hand, is a passive diagnostic that measures fusion-born neutrons escaping the plasma from the entire line-of-sight 33,34 . As the signal-to-noise ratio of FIDA measurements is typically superior close to the plasma center, where the fastion density is typically highest, we focus on central measurement volumes of the $\mathrm{O}$ - and B-port FIDA views at major radii $R \sim 196 \mathrm{~cm}$ and $R \sim 194 \mathrm{~cm}$ 
Table 1. Employed NBI sources during the studied discharges. The power fractions are given for each full-, half- and one-third energy component as $\left(P_{\text {full }}: P_{\text {half }}: P_{\text {third }}\right)$.

\begin{tabular}{|l|c|c|c|c|c|c|c|c|}
\hline & \multicolumn{2}{|c|}{$\# \mathbf{7 5 4 3 9}$} & \multicolumn{2}{c|}{ \# 75448 } & \multicolumn{2}{c|}{ \# 75455 } & \multicolumn{2}{|c|}{ \# 75469 } \\
\hline Beam & NBI1L & NBI1R & NBI1L & NBI2R & NBI1L & NBI2L & NBI1L & NBI2L \\
\hline Injection energy $[\mathrm{keV}]$ & 50 & 56 & 50 & 54 & 50 & 56 & 52 & 55 \\
\hline Power $[\mathrm{MW}]$ & 0.82 & 0.95 & 0.82 & 0.98 & 0.82 & 1.05 & 0.72 & 0.97 \\
\hline Power fractions & $70: 16: 14$ & $76: 13: 11$ & $70: 16: 14$ & $74: 18: 8$ & $70: 16: 14$ & $81: 11: 8$ & $69: 17: 14$ & $81: 11: 8$ \\
\hline
\end{tabular}
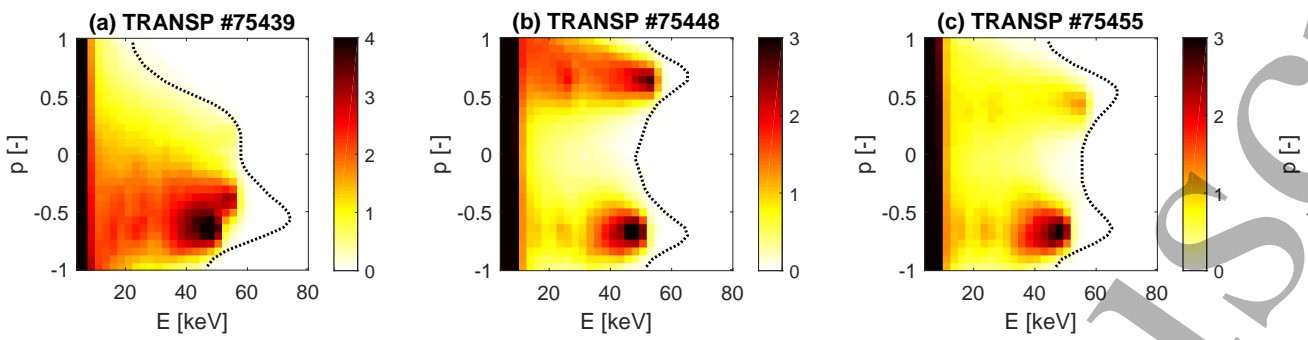

(d) TRANSP \#75469

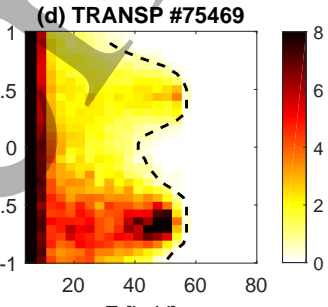

Figure 3. TRANSP distributions for discharges (a) \#75439, (b) \#75448, (c) \#75455 and (d) \#75469. The unit of the colorbar is $10^{16} \mathrm{keV}^{-1} \mathrm{~m}^{-3}$. In panels a-c, the dotted black lines separate the target areas from the null-measurement regions at higher energies as determined from measurements, whereas the dashed line in panel d indicates the largest fast-ion energies obtained at each pitch value as predicted by TRANSP.

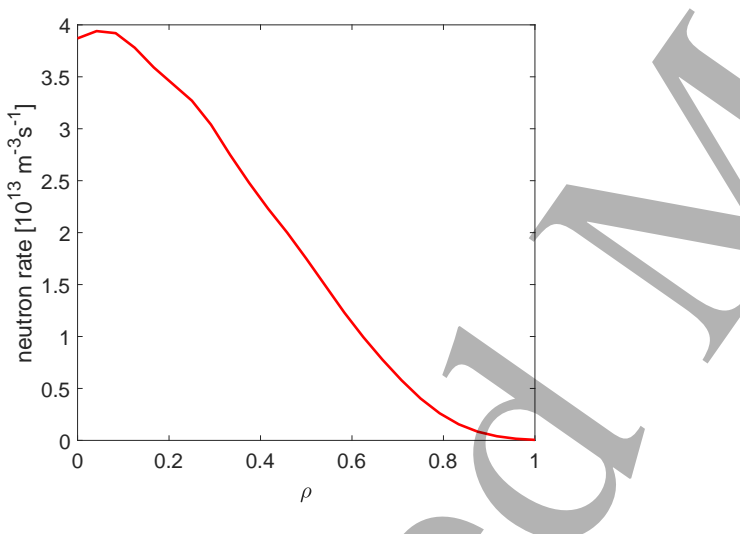

Figure 4. Neutron birth profile for neutrons born in beamtarget reactions simulated by the NUBEAM module in TRANSP plotted as a function of normalized minor radius $\rho$.

(normalized minor radius $\rho \sim 0.2$ ), respectively. These measurements have a spatial resolution of a few centimeters [17. For the EJ301 scintillator, we assume that the majority of the detected fusion-born neutrons are generated in beam-target reactions close to the magnetic axis as argued for in 33 . The predominant core-localization of neutron production is further supported by figure 4 that shows the neutron birth profile simulated by the NUBEAM module in TRANSP. The neutron birth rate is highest in the center as more fast ions reside in the plasma core than near the plasma edge. The slightly different locations of the FIDA and EJ301 measurements is not expected to have a strong effect on reconstructions based on combined FIDA and EJ301 measurements as spatial gradients are small close to the plasma center.

Figure 5 shows the absolutely calibrated signals measured by the two FIDA views. A notch filter with a width of $2 \mathrm{~nm}$ blocks the light around the unshifted D-alpha line at $656.1 \mathrm{~nm}$ in order to avoid saturation of the camera 42 . The instrumental broadening is estimated to be $0.1 \mathrm{~nm}$ for the Bport view and $0.33 \mathrm{~nm}$ for the O-port view [17]. Background radiation has been subtracted from the signals by beam modulation [40], and small residual off-sets, arising due to slightly imperfect background subtraction, have additionally been subtracted from the O-port signals. The measured signals are shown together with the signals predicted by the simulation code FIDASIM 43 45, including the FIDA, halo and beam emissions with applied instrumental broadening. For the vertical B-port signals, the beam emission feature is within the notch filter. Conversely for the O-port signal, the beam emission is in the red-shifted wing due to the tangential viewing geometry. This reduces the experimentally accessible spectral ranges that are reliable for fast-ion velocity-space tomography, but allows a comparison between the simulated and measured beam emission intensities. For all discharges, the intensity of the simulated O-port beam emission feature differs from the measured signals by a factor of two. This is possibly due to uncertainties in the absolute calibration of the signals or uncertainties in the assumed divergence of the NBI. In FIDAbased reconstructions of the fast-ion distribution, this disagreement translates to uncertainties in absolute values of solutions.

Both the red- and blue-shifted wings of the mea- 
sured O-port signals are well-modelled by FIDASIM for all discharges. However, especially the blue-shifted wings of the B-port measurements show discrepancies from the simulations. This might be caused by passive FIDA emission and is expected to cause discrepancies between measurement-based reconstructions and simulations especially at low energies according to the velocity-space sensitivities of measurements with small Doppler-shifts. Nevertheless, we choose to include these measurements in measurement-based reconstructions in order to retain sufficient velocity-space coverage and sensitivities.

In order to reliably reconstruct the dilute highenergy part of the ion distribution despite the strong signal caused by the high-density population at low energies, we use only the part of the spectra solely sensitive to ions with energies above $8 \mathrm{keV}$. This corresponds to spectral measurements with an absolute Doppler-shift of at least $\Delta \lambda \sim 1.9 \mathrm{~nm}$. We avoid also the parts of the spectra surrounding the carbon emission lines near $658 \mathrm{~nm}$ and the red-shifted beam emission in the O-port spectra. The resulting utilized spectral ranges are grey-shaded in figure 5. In the solely FIDA-based reconstructions in section 4 and section 5, parts of the spectra that are below the detection limit of the diagnostics are employed as nullmeasurements [20]. These are marked by hatches in figure 5. The velocity-space boundaries into the corresponding null-measurement regions are indicated by dotted lines in figure 3 a-c.

Figure 6 presents the measured EJ301 pulse height spectrum during discharge \#75469 expressed in units of kiloelectronvolt electron equivalent (keVee [33]). This spectrum relates to the fast-ion velocity distribution through instrument-specific EJ301 weight functions [34] and is shown together with the expected signal based on the neoclassical TRANSP distribution in figure $3 \mathrm{~d}$. The measurements are sampled over the four NBI1L blips in the time interval $3.5 \mathrm{~s}-7.5 \mathrm{~s}$, as marked by the four latter gray-shaded areas in figure 2b. This sampling method is employed in order to achieve a statistically significant measurement. From high-resolution neutron yield measurements during the discharge, the fast-ion slowing-down time is estimated to be $\sim 10 \mathrm{~ms}$. EJ301 measurements obtained during the first $10 \mathrm{~ms}$ after turning on NBI1L are, therefore, omitted in the analysis. Since EJ301 ultimately measures an averaged fast-ion distribution during the four NBI1L blips in the time interval $3.5 \mathrm{~s}-$ $7.5 \mathrm{~s}$, the FIDA spectra obtained during discharge \#75469 are, likewise, averaged over NBI1L blibs in times without ELM contamination.

The EJ301 compact single-plate scintillator has good capability of neutron/gamma discrimination 33, 46. However, the absolute calibration of the
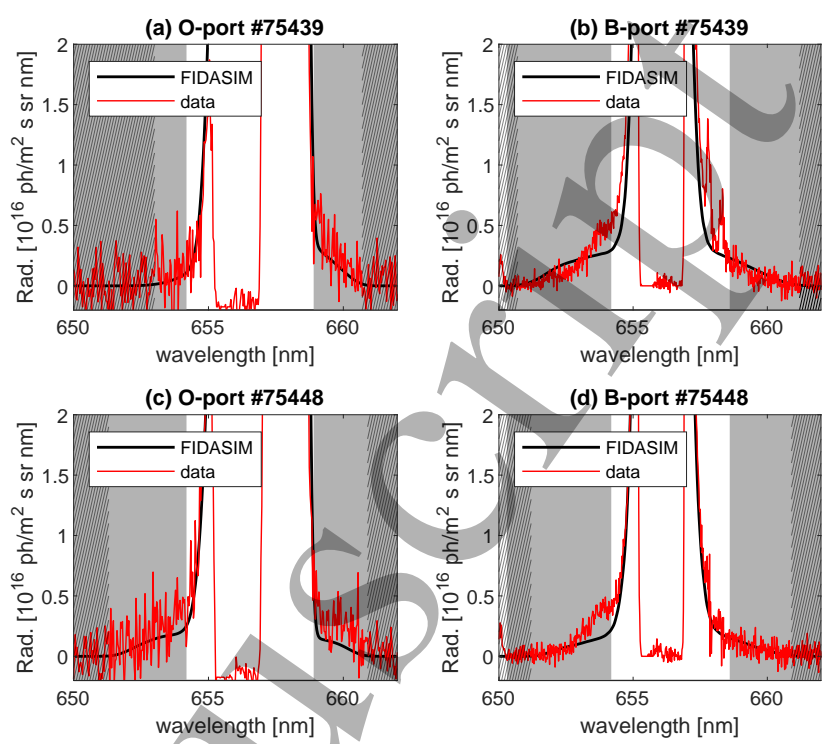

(e) O-port \#75455
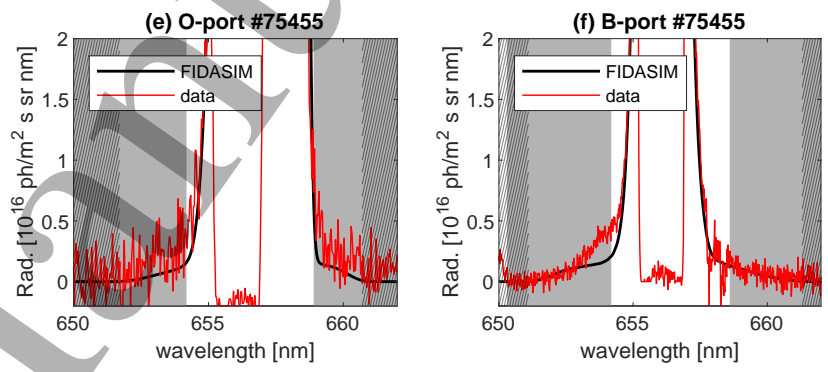

(g) O-port \#75469
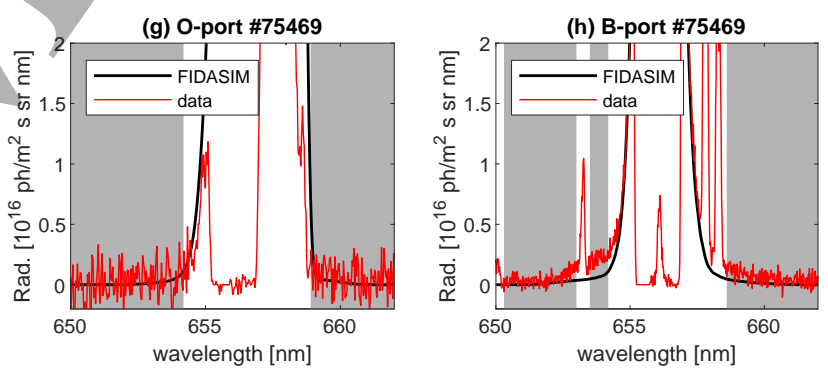

Figure 5. Absolutely calibrated measurements and simulated signals from the FIDA O- and B-ports at $R \sim 195 \mathrm{~cm}$ and $t=4.2 \mathrm{~s}$ during discharges (a-b) \#75439, (c-d) \#75448 and (e-f) \#75455 as well as at $t=6.2 \mathrm{~s}$ during discharge (g-h) \#75469. The experimentally reliable measurements lie within the greyshaded areas. In panels a-f, null-measurements are marked by hatches.

EJ301 measurements remains uncertain. In combined tomography from different diagnostic methods (here NES and FIDA), good relative calibration of the spectra is paramount. As the absolute calibration of the EJ301 scintillator is uncertain, we calibrate both the FIDA and EJ301 measurements to signals based on the TRANSP distribution (figure 3 d). In order to retain the relative calibration between the O- and B-port FIDA views, we apply only a single calibration factor to the signals from each of the FIDA views. This calibration has been applied only to 


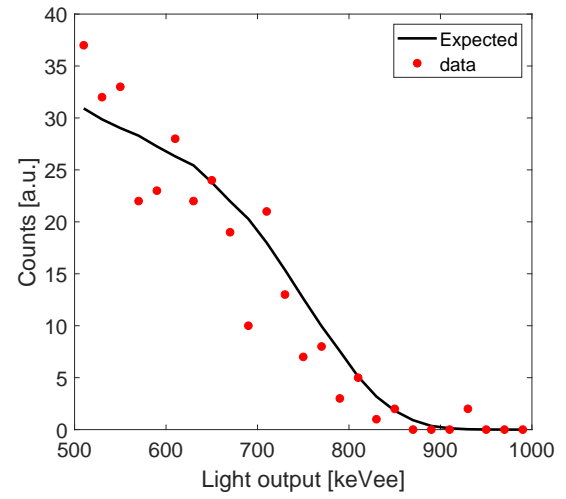

Figure 6. Neoclassically expected and experimentally measured EJ301 pulse high spectrum during dual-beam injection in discharge \#75469.
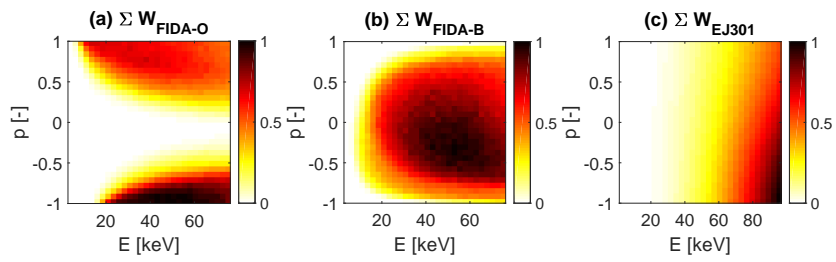

Figure 7. Weight function coverages of the (a) O-port (FIDAO) and (b) B-port (FIDA-B) FIDA views as well as (c) the EJ301 scintillator computed as the sum of all weight functions for the experimentally available spectral ranges.

measurements obtained during discharge \#75469. For discharge \#75469, we, therefore, make no statements about absolute values for reconstructions based on combined FIDA and EJ301 measurements.

\subsection{Weight functions}

The velocity-space sensitivities of measurements are determined by weight functions $w 22,23$ that relate the measured signal $s$ to the distribution $f$, through

$s=\int_{-1}^{1} \int_{0}^{\infty} w f d E d p$.

Weight functions are key to understand the measured signal and allow reconstructions of the distribution as they link fast-ion yelocities to signals in specific spectral ranges 22]. The sum of all weight functions reveals which regions in velocity-space are observed by the diagnostic 16]. This is shown in figure 7 for FIDA and EJ301 utilizing only the experimentally reliable spectral ranges. Whereas FIDA is sensitive to a large energy range in the target region, EJ301 significantly increases its sensitivity at energies close to and above the highest beam injection energies.

Previous tomographic studies on fast-ion velocity distributions have shown that multiple-view coverage in the target region is essential for providing reliable reconstructions using standard tomographic approaches $7,14,16$. Especially at low energies and small absolute values of the pitch, this is not fulfilled for the solely two-view FIDA setup installed at EAST. However, as the largest fast-ion densities are expected to be at greater absolute pitch values (figure 3), reconstructions can still be obtained keeping in mind that regions with single-view coverage are less reliable than regions with dual-view coverage as argued for in 15 .

The reconstructions can additionally be aided by changing the tomographic approach. In the following section, this is pursued by expressing the distribution in a basis relying on the physically expected functional shape of the distribution.

\section{Slowing-down basis functions in tomographic problems}

Throughout the physical sciences, a common approach to improve the solution of tomographic problems is to expand the solution in a set of basis functions [47]. Here we adopt this approach by employing a set of basis functions relying on the neoclassically expected slowing down of ions in a fusion plasma. This basis is chosen in order to reflect the expected nature of the distribution. Note, however, that many other choices of basis functions will also work and might prove beneficial or even superior in later studies.

In a steady-state scenario with neoclassical transport, a fast ion with a specific energy and pitch will change its velocity through Coulomb collisions. At high energies, the energy is transferred predominantly to electrons causing little pitch-angle scattering. Collisions with ions dominate below the crossover speed 39]. For the EAST discharges, this is evaluated to be $\sim 26 \mathrm{keV}$ for deuterium. As estimates for the collision-caused slowing down of ions described by a steady-state distribution, we use the analytical expressions derived in 48, 49]. Owing to possible non-neoclassical transport and uncertainties in the crossover speed, we further dampen the slowingdown functions farther from the birth velocity by the complementary error function. A selection of resulting slowing-down functions is shown in figure 8. These functions are contained in the full slowing-down basis used in section 4 , section 5 and section 6 .

In this work, we create the slowing-down basis such that for each pixel in the pixel basis, the slowingdown basis contains one error-function-dampened slowing-down function with birth energy and pitch at that pixel. The pixel and slowing-down bases, hence, contain the same number of basis functions, allowing the slowing-down basis to span the entire pixel space.

A change-of-variables to the slowing-down basis 

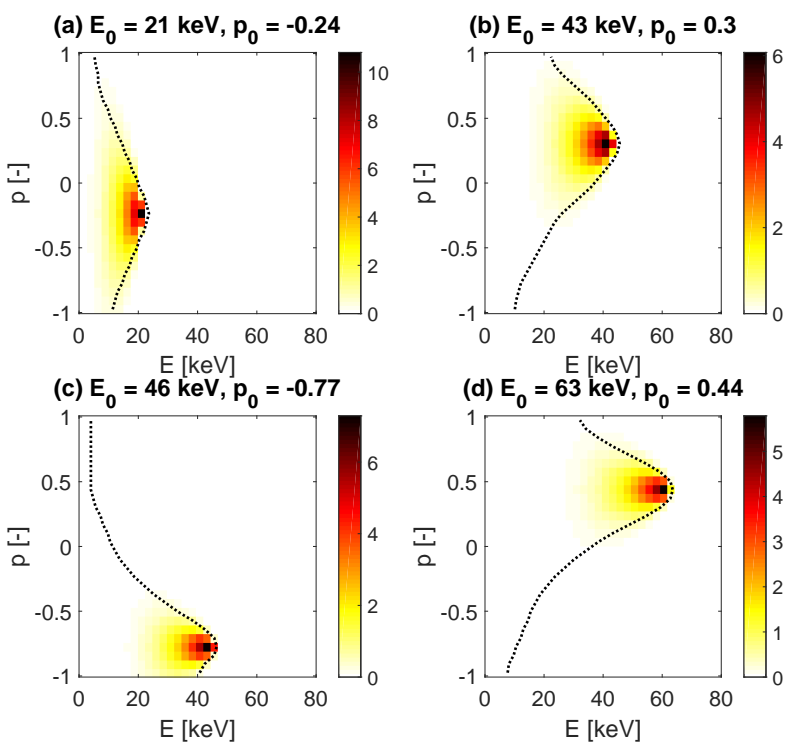

Figure 8. Selection of normalized slowing-down basis functions. The dotted lines indicate the shape of the slowing-down functions before dampening by the error function.

can be introduced in tomographic problems by reformulating the forward problem. To do so, assume that the distribution $f$ is the sum of $k$ slowing-down functions:

$f=\sum_{i=1}^{k} a_{i} f_{S D, i}$

where $\left(f_{S D, 1}, \ldots, f_{S D, k}\right)$ detones the slowing-down basis, whilst $\left(a_{1}, \ldots, a_{k}\right)$ is the set of coefficients that computes $f$ given the basis. The forward problem (1) then takes the form

$$
\begin{aligned}
s & =\int_{-1}^{1} \int_{0}^{\infty} w \sum_{i=1}^{k} a_{i} f_{S D, i} d E d p \\
& =\sum_{i=1}^{k} a_{i} \int_{-1}^{1} \int_{0}^{\infty} w f_{S D, i} d E d p .
\end{aligned}
$$

Consider now the discretization of (1):

$S=W F$.

Here $S$ is the vector containing the measurements, $F$ is the vector containing the distribution, and $W$ is the matrix containing the weight functions 7 . Introduce now the matrix $F_{S D}$ with columns given by the discretized slowing-down functions in energy and pitch and the vector $A=\left[a_{1}, \ldots, a_{k}\right]^{T}$ such that

$F=F_{S D} A$.

Then the discretized form of (4) can be written as $S=W F_{S D} A=W_{S D} A$

where $W_{S D}=W F_{S D}$. Note that $W_{S D}$ does not directly link the energy-pitch location of a fast ion to the generated signal, but rather gives information about which slowing-down distributions can cause a signal in a specific wavelength range. A comparison of $W$ and $W_{S D}$ is given in figure 9 for a number of spectral ranges. The outer shapes of the weight functions are not significantly affected by the change of basis. On the other hand, the inner structure of $W_{S D}$ is noticeably less fine-structured than the inner structure of $W$. This is especially prominent at the low-energy border of the weight functions. Here the pixel-based weight functions $W$ have a sharp sensitivity increase that often causes artefacts in reconstructions from sparse FIDA measurements. This indicates that applying the slowing-down basis can ultimately provide a smoothing of the reconstructions, which is not always attainable from pixel-based reconstruction methods.

\section{Inversions of synthetic FIDA signals in various bases}

Fast-ion velocity-space tomography determines the most likely fast-ion velocity distribution from a collection of signals. This is essentially done by finding $F$ from $S$ and $W$ in (4). However, this problem is ill-posed and must be regularized in order to provide a stable solution [13. Here, we use the zeroth-order Tikhonov method where solution norms are penalized, as it has been found to provide better results for the two-view FIDA system at EAST [19] than the more commonly used first-order Tikhonov method that penalizes large gradients in energy and pitch $[13$. We additionally impose a non-negativity constraint [20] on the solution. In the standard pixel-basis, the nonnegative zeroth-order Tikhonov solution is found as

$$
\begin{gathered}
F^{*}=\min _{F}\left\|\left(\begin{array}{c}
W \\
\lambda I
\end{array}\right) F-\left(\begin{array}{c}
S \\
0
\end{array}\right)\right\|_{2} \\
\text { subject to } F \geq 0
\end{gathered}
$$

where $I$ is the identity matrix and $\lambda$ is the regularization strength. In the slowing-down basis we instead seek to invert (6), i.e. we seek the vector $A$ holding the basis-function expansion coefficients $\left(a_{1}, \ldots, a_{k}\right)$. This is also an ill-posed problem that we likewise regularize by a zeroth-order Tikhonov penalty term:

$$
\begin{aligned}
A^{* *}= & \min _{A}\left\|\left(\begin{array}{c}
W_{S D} \\
\lambda I
\end{array}\right) A-\left(\begin{array}{c}
S \\
0
\end{array}\right)\right\|_{2} \\
& \text { subject to } F_{S D} A \geq 0
\end{aligned}
$$

such that the reconstructed distribution is $F^{* *}=$ $F_{S D} A^{* *}$ according to (5). The constraint in (8) expresses non-negativity of $F$. For reconstructions computed from both (7) and (8), we normalize the forward problem by the measurement uncertainty 8 , and include null-measurements as a constraint 20]. We apply null-measurements as a constraint rather than a 

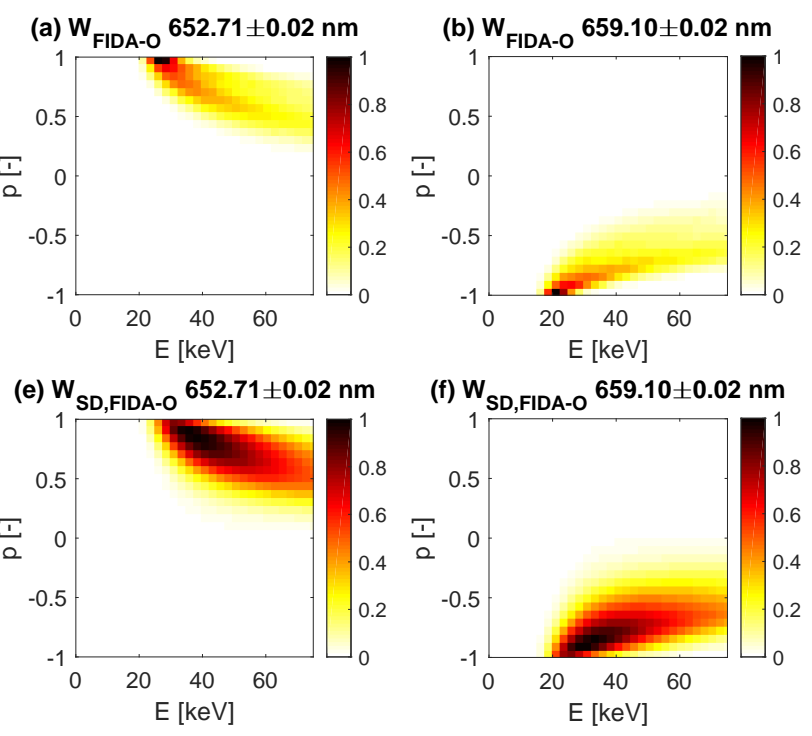
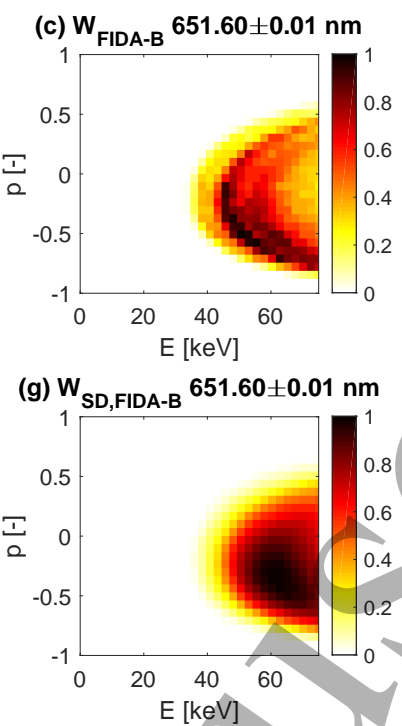

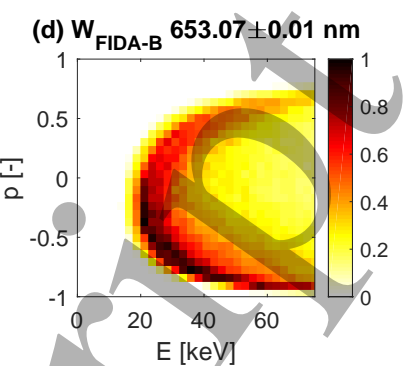

(h) $\mathrm{W}_{\mathrm{SD}, \mathrm{FIDA}-\mathrm{B}} 653.07 \pm 0.01 \mathrm{~nm}$

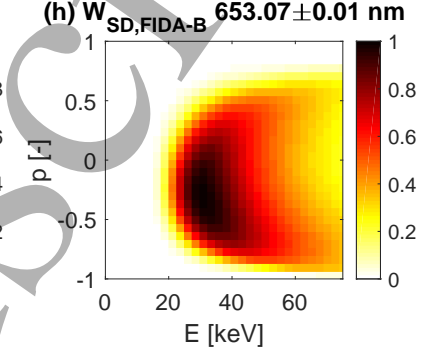

Figure 9. Selection of weight functions (a-d) in the pixel basis $(W)$, and (e-h) in the slowing-down basis ( $\left.W_{S D}\right)$ for the O- and B-port FIDA views. The weight functions are shown for the measured spectral resolution and each weight function is normalized by its own maximum value.

penalty in the null-measurement region 14 in order to highlight the effect of changing the basis.

If the basis matrix $F_{S D}$ is invertible, the change of basis can be interpreted as a modification of the regularizing term. This is evident when introducing the inverse of (5),

$A=F_{S D}^{-1} F$

in (8):

$$
\begin{aligned}
F^{* *}= & \min _{F}\left\|\left(\begin{array}{c}
W \\
\lambda F_{S D}^{-1}
\end{array}\right) F-\left(\begin{array}{c}
S \\
0
\end{array}\right)\right\|_{2} \\
& \text { subject to } F \geq 0
\end{aligned}
$$

Equation 10 allows a direct computation of the reconstructed distribution $F^{* *}$, but relies on the existence of $F_{S D}^{-1}$. Mathematically, (9) suggests that the expansion of the velocity distribution into basis functions is equivalent to a coordinate transformation [50]. In the inverse problem literature, this is referred to as a standard-form transformation. The longrange correlation given by the basis functions can be quantified and visualized through the covariance matrix $C$. The inverse of $C$ appears as the prior in the Bayesian picture 31 . When the invertible basis $F_{S D}$ acts as the only imposed regularization, as in $(10)$, the covariance matrix can be determined as $C=\left(\lambda^{2} F_{S D}^{-T} F_{S D}^{-1}\right)^{-1}=\lambda^{-2} F_{S D} F_{S D}^{T}$.

Examples of entries in $C$ are shown in figure 10 . From these, it is evident that the covariance matrix ensures smoothness by correlating a given pixel to the pixels in its vicinity. The correlation decreases with increasing distance in $(E, p)$-space, with a pattern depending
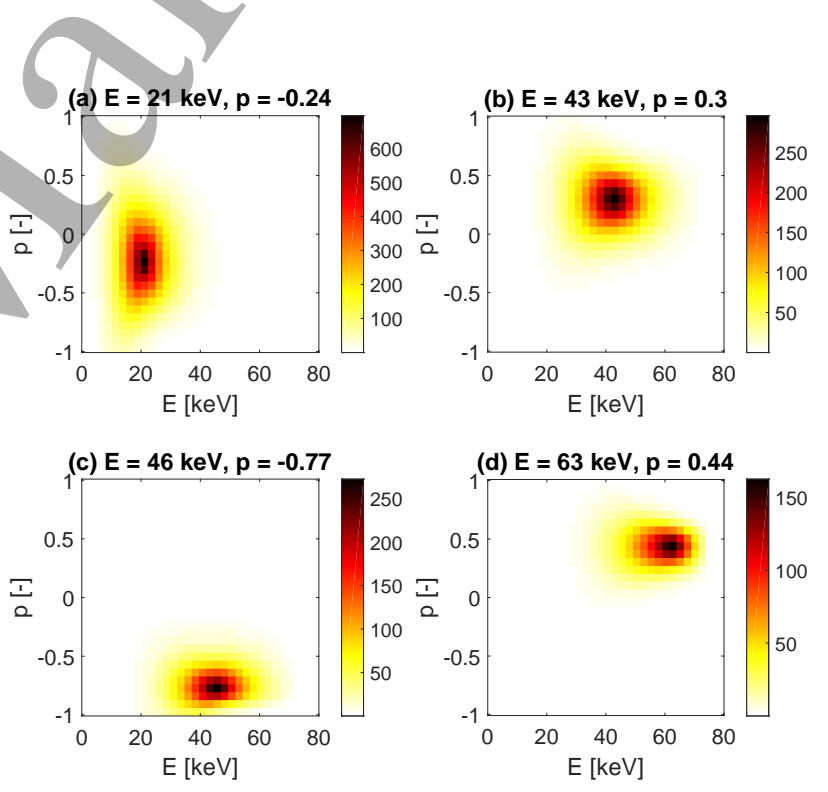

Figure 10. Examples of entries in the covariance matrix $C$ when the invertible slowing-down basis determines the regularization.

on the position in $(E, p)$-space due to the physics of the slowing-down process: For low energies, where ion collisions dominate, the correlation is ensured over a large pitch range. For high energies, where collisions with electrons dominate, the correlation is rather narrow in pitch. The slowing-down process is thus encoded in the regularization of our tomography problem. This long-range correlation of pixels in the reconstructions is reasonable to include in the analysis of the discharges studied in this work, as transport in 
these discharges are expected to be neoclassical.

If an arbitrary regularizer $L$ is used rather than the zeroth-order Tikhonov regularizer $I$ in (8), (10) takes the form

$$
\begin{aligned}
F^{* *}= & \min _{F}\left\|\left(\begin{array}{c}
W \\
\lambda L F_{S D}^{-1}
\end{array}\right) F-\left(\begin{array}{c}
S \\
0
\end{array}\right)\right\|_{2} \\
& \text { subject to } F \geq 0 .
\end{aligned}
$$

Here, the full regularizer is $L F_{S D}^{-1}$. However, as the chosen basis functions provide a smoothness to the solution, it is often unnessecary to include an additional regularizer $L$ in order to obtain smooth solutions. We, hence, employ only the basis functions as regularization in the following. Additionally, note that since (8) and $(10)$ are equivalent, they provide identical solutions within the expected numerical accuracy. As the conditioning number of $F_{S D}$ can be quite large, computations are often more stable when using (8) rather than (10). For basis function expansion reconstructions we will, therefore, show only results computed with (8).

For the EAST two-view FIDA system, the differences in reconstructions based on (7) and 8 are shown in figure 11 for the three reference discharges. Here, synthetic signals based on the TRANSP simulations (figure $3 \mathrm{a}-\mathrm{c}$ ) were generated in the experimentally reliable spectral ranges. For each spectrum, 10\% Gaussian noise was added. The regularization strength for each of the reconstructions based on these synthetic signals is chosen as the one that produces the smallest two-norm of the pixeldifferences between the reconstruction and the true solution for $E>20 \mathrm{keV}$. Both approaches succeed in locating regions of high densities. However, the reconstructions in the slowing-down basis (figure $11 \mathrm{~d}-$ f) perform better than the pixel reconstructions in especially two respects: Firstly, they provide smoother solutions as a consequence of the long-range correlation following the expansion in basis functions. Secondly, they manage to more accurately determine the fastion birth velocities.

\section{FIDA measurement-based reconstructions}

Also the reconstructions from FIDA measurements in figure 12 find that the solutions using slowingdown basis functions (figure $12 \mathrm{~d}-\mathrm{f}$ ) are smoother than the pixel reconstructions (figure $12 \mathrm{a}-\mathrm{c}$ ). Within the measurement uncertainty, forward-modelled spectra based on the reconstructed distributions in figure 12 agree well with the measurements in figure $5 \mathrm{p}$-f. At small Doppler-shifts, forward-modelled signals based on the slowing-down reconstructions tend to follow the measurements more accurately than forward-modelled signals based on the pixel reconstructions. This is due to the large densities at $E<25 \mathrm{keV}$ that appear in the slowing-down reconstructions but not in the pixel reconstructions. Recall, however, that the exact origin of the disagreement between measurements and simulation at small blue-shifts in the B-port signals (figure 5) is still uncertain. Hence, a better agreement at the smallest accessible Doppler-shifts between measurements and forward-modelled signals based on reconstructions does not necessarily provide a better reflection of the physics at play.)

As for the synthetic case, the pixel-norm regularizing approach tends to create solutions with shapes dictated by the boundary of the null-measurement region. This results in densities significantly above the injection energy. As similar features are observed in reconstructions based on synthetic signals, where the true solution is known, these high-energy densities are likely artefacts caused by the limited weight function coverage, measurement noise and a consequently limited velocity-space resolution.

Despite the limited velocity-space resolution, the reconstructions from measurements clearly distinguish the differences in birth pitch of fast ions generated from different NBI sources. This is especially evident from figure 13 that gives the velocity-space resolved pixel differences between discharges \#75439 and \#75455 as well as between discharges \#75448 and \#75455 as calculated by TRANSP and from measurementbased reconstructions. From figure $13 \mathrm{~b}$, it is evident that discharge \#75439 has an excess of ions with negative pitches compared to \#75455 due to NBI1R heating, whereas the NBI2L heating in discharge \#75455 creates a surplus of ions born with pitches close to 0.5 compared to discharge \#75439, as also predicted by TRANSP. Likewise in figure 13, the larger pitch values of ions born from NBI2R in discharge \#75448 compared to ions born from NBI2L in discharge \#75455 is clearly evident.

\section{Integrated data analysis of FIDA and NES spectra}

In this section, we illustrate some of the benefits and limitations of combined FIDA and NES tomography using measurements obtained during discharge \#75469. We employ the O- and B-port FIDA views using the same geometry as for the discharges studied in section 5, as well as EJ301 measurements given in figure 6 .

Figure 14 shows reconstructions for discharge \#75469 both from solely FIDA measurements (panels $\mathrm{a}$ and c) and from combined FIDA and EJ301 measurements (panels b and d). For both datasets, we present reconstructions calculated in the pixel basis (panels a and b) and in the slowing-down basis (panels c and d). We employ a non-negativity constraint on the 

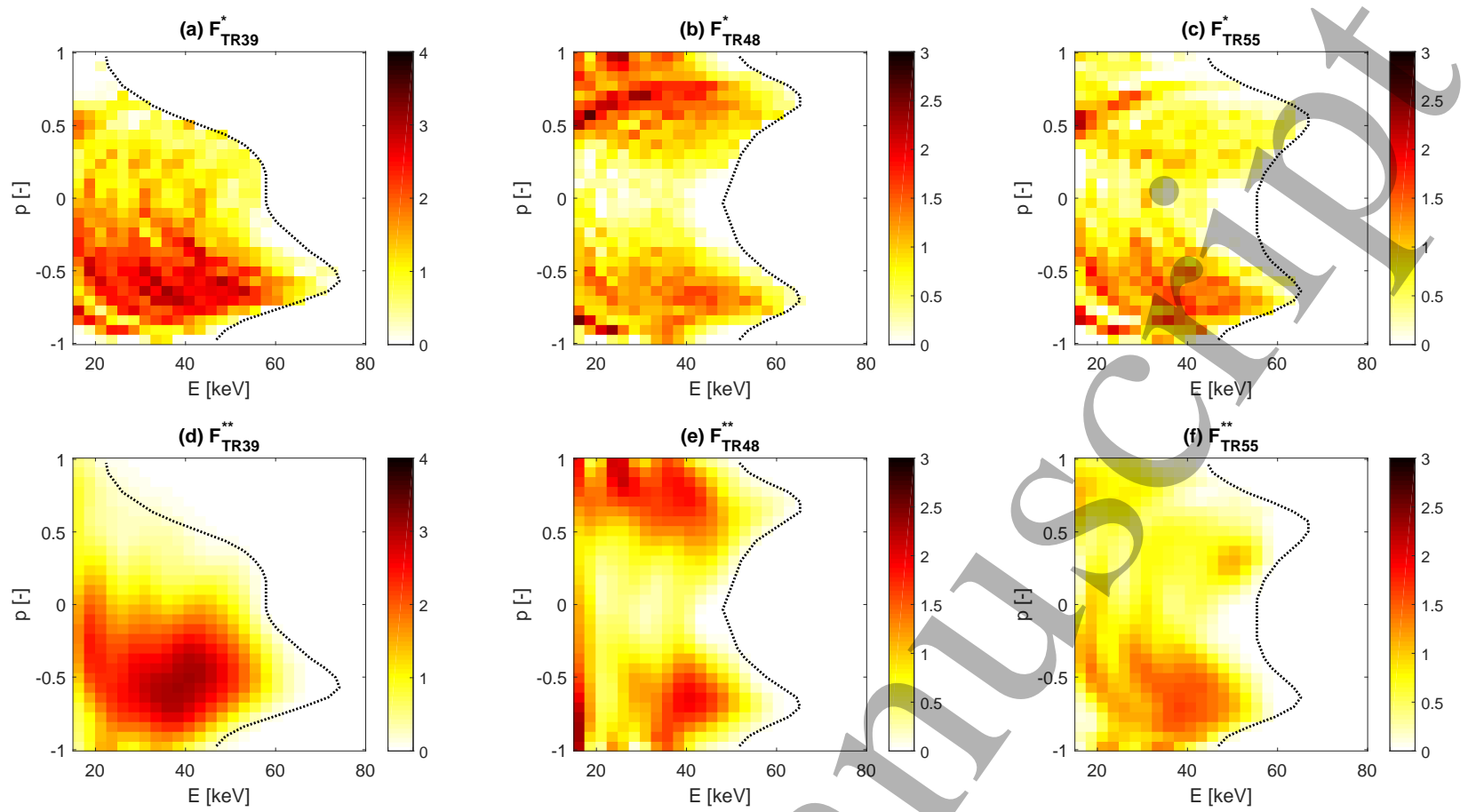

Figure 11. Reconstructions from a synthetic signal based on the TRANSP distributions for EAST discharges \#75439, \#75448 and \#75455 using (a-c) the zeroth-order Tikhonov method acting on pixels of the solution, and (d-f) the zeroth-order Tikhonov method acting on expansion coefficients in the slowing-down basis. The colormap unit is the same as in figure 3 and the dotted lines separate the target areas from the null-measurement regions at higher energies.
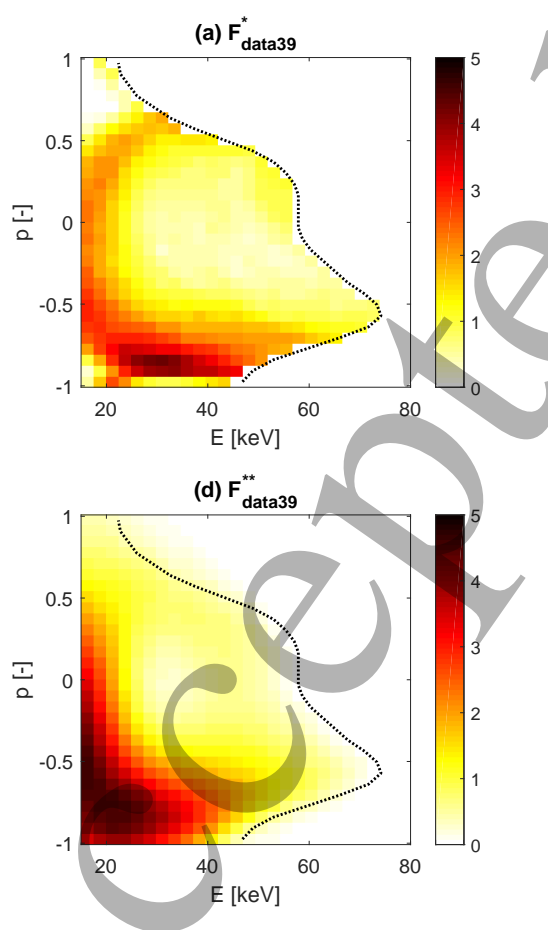
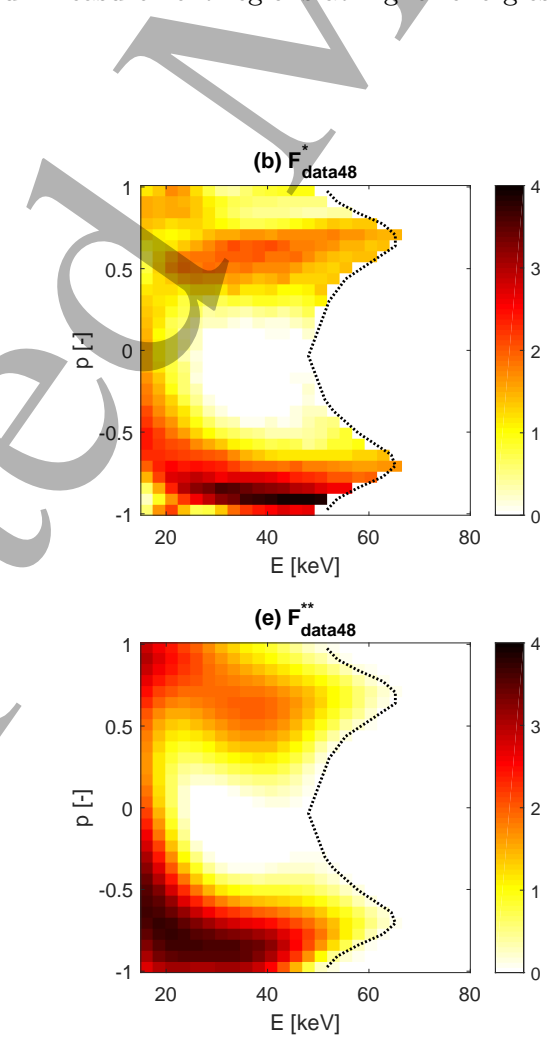
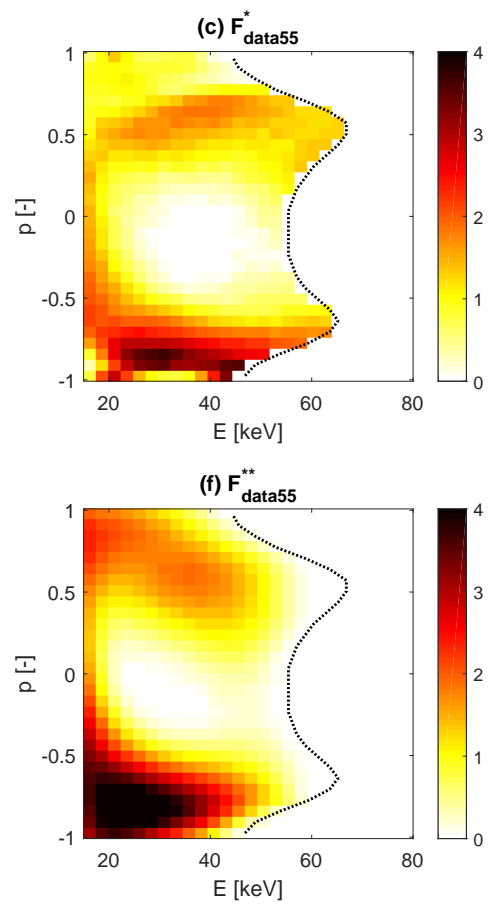

Figure 12. Reconstructions from measurements. The notation, unit and layout are the same as in figure 11 and the dotted lines separate the target areas from the null-measurement regions at higher energies. 

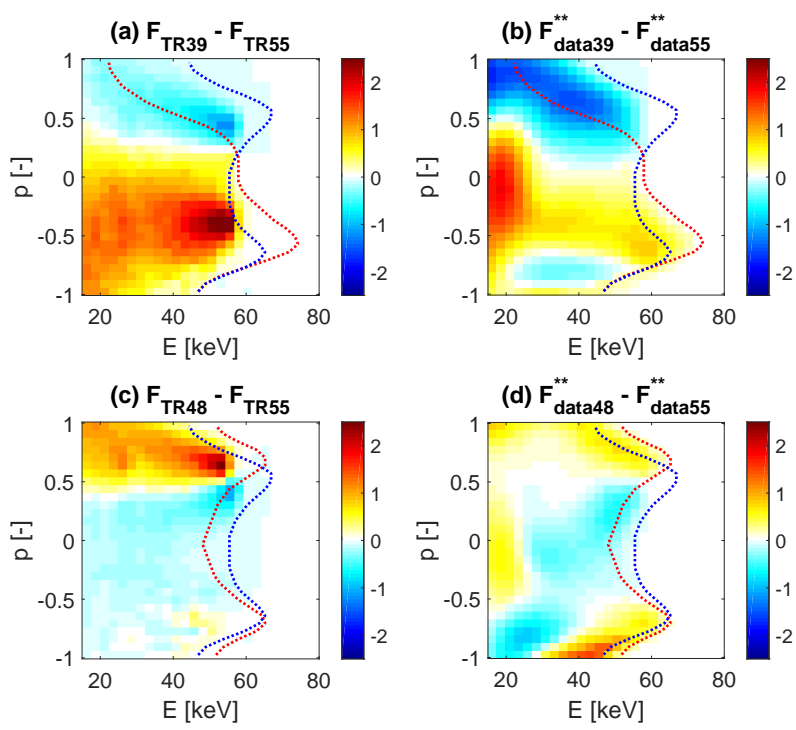

Figure 13. Velocity-space resolved pixel differences between the fast-ion distributions during (a-b) discharges \#75439 and \#75455 and (c-d) discharges \#75448 and \#75455 as computed by TRANSP and from slowing-down reconstructions from measurement. The dotted lines mark the boundaries to the nullmeasurement regions for \#75439 (red) and \#75455 (blue) in the upper panel and for \#75448 (red) and \#75455 (blue) in the lower panel. Same notation and unit as in figure 3 and figure 12
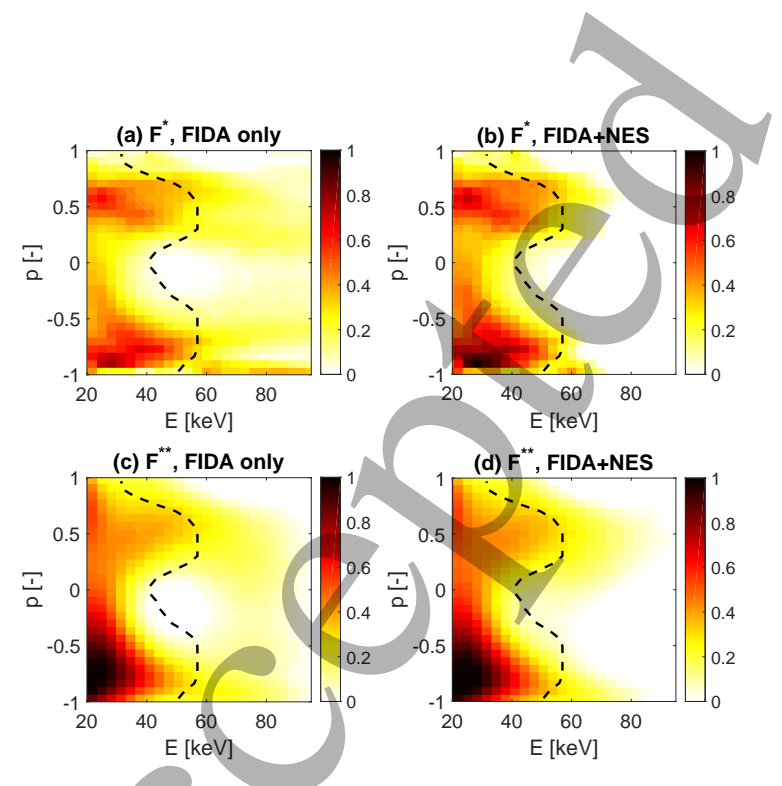

Figure 14. Reconstructions of the fast-ion velocity distribution during EAST discharge \#75469 using (a-b) the pixel basis and (c-d) the slowing-down basis employing only FIDA measurements and combined FIDA and EJ301 measurements. All panels are scaled by the same factor. As in figure $3 \mathrm{~d}$, the dashed lines indicates the largest fast-ion energies obtained at each pitch value as predicted by TRANSP. distribution, but do not include null-measurements in order to allow the measurements to directly determine the density distribution above the full injection energy of the neutral beam. In both tomographic approaches, the reconstructions from measurements reveal the two-beam slowing-down distribution created by simultaneous NBI1L and NBI2L injection.

At energies above the full injection energy, the pixel-based reconstruction from solely FIDA measurements is plagued by artefacts that also appear in reconstructions from noisy synthetic two-view FIDA signals based on the TRANSP distribution. Including the EJ301 signal together with the FIDA measurements strongly improves the reconstruction at these energies, even though the EJ301 measurement are relatively sparse. This is an effect of the low neutron count rates (figure 6) that indicate that only few ions have velocities in the region covered by the EJ301 scintillator which is predominantly above the neutral beam injection energies. Hence, in this case the EJ301 signal ultimately serves as null-measurements allowing suppression of artifacts in the null-measurement region without including a dedicated penalty or restriction on the solution in this region. This effect is less pronounced when reconstructing in the slowing-down basis (panel c and d). Here the inclusion of EJ301 measurements only slightly improves the reconstruction at negative pitches for $E \sim 60-80 \mathrm{keV}$ without significantly affecting the reconstruction at lower energies. The reason for the different benefits gained from including EJ301 measurements in the pixel and slowing-down approaches is still uncertain. The energy-dependence of the benefits, on the other hand, can be explained by the velocity-space sensitivities of the diagnostics: during this discharge, fast deuterium ions are created purely by neutral beam injection at energies close to the lower energy limit of the EJ301 sensitivity range. The EJ301, therefore, serves as a third diagnostic view that in combination with the dual-view FIDA setup provides multiple-view coverage for all pitches at high energies. This improves the reconstructions in the high-energy range, but does not necessarily strongly affect the solution elsewhere.

\section{Moments of the distribution function}

Taking the moments of the distributions allows estimations of the fast-ion densities, pressures and current densities 10. In this section, we compute absolute values from the FIDA-based reconstructions in section 5 with the purpose of evaluating the level of agreement between reconstructions in the two bases as well as to quantitatively compare measurement-based reconstructions and predicted TRANSP distributions. 
This is followed by a discussing on moments from the FIDA+NES reconstructions in section 6. We, however, restrain from including absolute values based on FIDA+NES reconstructions, as the FIDA and NES spectra are scaled to expected signals for discharge \#75469 due to the uncertainty in the absolute calibration of the EJ301 spectrum. With a better resolution and greater velocity-space coverage of the low-energy range of velocity space, also the bulk deuterium densities, pressures, currents and temperatures could be directly calculated from the reconstructed ion distribution. However, currently this is not possible from FIDA measurements at EAST.

The kinetic pressure can be determined as the trace of the pressure tensor, i.e. the second moment of the distribution. This yields

$$
\begin{aligned}
P_{i}= & \frac{2}{3} \int_{-1}^{1} \int_{0}^{\infty}\left(E-v_{d i \|} p \sqrt{2 m_{i} E}+\frac{1}{2} m_{i} v_{d i \|}^{2}\right) \\
& f(E, p) \mathrm{d} E \mathrm{~d} p
\end{aligned}
$$

for rotational symmetry about the magnetic field. Here the drift velocity is assumed to be fully parallel to the magnetic field and, hence, given by

$$
v_{d i \|}=\frac{1}{n_{i}} \int_{-1}^{1} \int_{0}^{\infty} p \sqrt{\frac{2 E}{m_{i}}} f(E, p) \mathrm{d} E \mathrm{~d} p
$$

where $n_{i}$ is the fast-ion density computed as the zeroth moment of the distribution. From the drift velocity and density, the fast-ion current density can be computed as

$j_{i \|}=q n_{i} v_{d i \|}=q \int_{-1}^{1} \int_{0}^{\infty} p \sqrt{\frac{2 E}{m_{i}}} f(E, p) \mathrm{d} E \mathrm{~d} p$

where $q$ is the fast-ion charge. The resulting values for the measurement-based reconstructions relative to the expected TRANSP values are summarized in table 2 for fast ions with energies above $20 \mathrm{keV}$. The results are consistent for measurement-based reconstructions using both of the tomographic approaches.

For the purely co-current injection in discharge \#75439, the reconstructed density, pressure and current density are well-modelled by TRANSP. On the other hand, for simultaneous co- and counter-current injection in discharges \#75448 and \#75455, discrepancies between reconstructed values and simulations are present, with reconstructed densities and pressures exceeding the TRANSP values by up to $70 \%$. The larger densities in reconstructions compared to the TRANSP predictions is partly explained by the strong B-port signals that exceeds the FIDASIM simulation in the spectral range 653-654 nm (figure 5). Other important factors that can cause discrepancies between simulation and reconstructions are uncertain absolute calibration of measurements as well as the complex structure of distributions consisting of ions born simultaneously at both positive and negative pitches as these are more challenging to reconstruct from sparse datasets than distributions that are strongly lopsided towards either positive or negative pitches.

For discharge \#75455, the reconstructed fastion current density is well-modelled by TRANSP. For discharge \#75448, on the other hand, the lack of fast-ion current density suggested by TRANSP is not observed in reconstructions from measurements. The large apparent discrepancy in the TRANSPpredicted and reconstructed current density for discharge \#75448 is partly explained by division by a small denominator. A more reasonable comparison between the reconstructed and simulated current density for discharge \#75448 can be obtained by instead computing the current densities driven solely by either positive- or negative-pitch fast ions: $j_{i \|, \text { data } 48}^{+} / j_{i \|, T R 48}^{+} \sim 1.2$ for positive-pitch fast ions and $j_{i \|, \text { data } 48}^{-} / j_{i \|, T R 48}^{-} \sim 1.9$ for negative-pitch fast ions. Both of these numbers are close to the relative difference between the reconstructed and simulated density. It is worth noticing that the best agreement between reconstruction and simulation is obtained for positive-pitch fast ions. This is explained again by the large B-port signal between $653 \mathrm{~nm}$ and $654 \mathrm{~nm}$ that mostly affect the inferred fast-ion densities of the negative-pitch population as measurements in this/spectral range is predominantly origining from negative-pitch fast ions.

For discharge \#75469, we scale the NES and FIDA spectra to the expected signals based on the TRANSP distribution. As a result of that, the reconstructed densities are within $10 \%$ of the TRANSP density for $E>20 \mathrm{keV}$ for all reconstructions presented in section 6. This agrees with the uncertainty in moments of reconstructed distributions based on noisy synthetic signals. The reconstructed pressure exceeds the TRANSP prediction by $30-40 \%$ in the FIDA-only reconstructions. In both the pixel and slowing-down reconstructions based on combined FIDA and NES measurements, the reconstructed pressure agrees within a few percent with the pressure derived from TRANSP. The disagreement between predicted and reconstructed pressure in the FIDAonly reconstructions is, hence, expected to be caused predominantly by the artefacts at high energies that are avoided when including measurements from the EJ301 scintillator. The reconstructed current densities, on the other hand, are only $20-45 \%$ of the predicted value for all inversions. This is explained partly by a disagreement between the distribution of ions about the line $p=0$ in reconstructions compared to TRANSP. From the TRANSP simulation the distribution is expected to be strongly asymmetric about the line $p=0$. The ratio of fast-ion densities 
Table 2. Absolute densities $n_{i}$, pressures $P_{i}$ and current densities $j_{i \|}$ for fast ions with energies above $20 \mathrm{keV}$ from the TRANSP distribution $\left(F_{T R}\right)$ and relative values to TRANSP for measurement-based reconstructions using either the pixel basis $\left(F^{*}\right)$ or the slowing-down basis $\left(F^{* *}\right)$. The sign of $j_{i \|}$ is positive in the direction of the plasma current. For reconstructions from synthetic signals with $10 \%$ Gaussian signal noise plus a Gaussian background noise of $10 \%$ of the averaged bremstrahlung level, the derived quantities, $n_{i}, P_{i}$ and $j_{i \|}$, lie mostly within $10 \%$ of the true values for $E>20 \mathrm{keV}$.

\begin{tabular}{|c|c|c|c|c|c|c|c|c|c|}
\hline & \multicolumn{2}{|c|}{$\# \mathbf{7 5 4 3 9}$} & \multicolumn{2}{c|}{ \# 75448 } & \multicolumn{3}{c|}{ \# 75455 } \\
\hline & $F_{T R}$ & $F^{*}$ & $F^{* *}$ & $F_{T R}$ & $F^{*}$ & $F^{* *}$ & $F_{T R}$ & $F^{*}$ & $F^{* *}$ \\
\hline$n_{i}$ & $8.2 \times 10^{17} \mathrm{~m}^{-3}$ & 0.9 & 0.9 & $5.5 \times 10^{17} \mathrm{~m}^{-3}$ & 1.4 & 1.4 & $4.6 \times 10^{17} \mathrm{~m}^{-3}$ & 1.6 & 1.7 \\
\hline$P_{i}$ & $2.9 \mathrm{kPa}$ & 0.9 & 0.8 & $2.2 \mathrm{kPa}^{2}$ & 1.4 & 1.3 & $1.8 \mathrm{kPa}^{-3}$ & 1.7 & 1.6 \\
\hline$j_{i \|}$ & $91 \mathrm{Am}^{-2}$ & 0.9 & 1.0 & $-7.4 \mathrm{Am}^{-2}$ & -3.7 & -3.6 & $25 \mathrm{Am}^{-2}$ & 1.0 & 1.2 \\
\hline
\end{tabular}

for $p<0$ and $p>0$ is $n^{-} / n^{+}=2.3$ for energies between $20 \mathrm{keV}$ and $60 \mathrm{keV}$. Such a strong asymmetry is not detected in the pixel reconstructions based on either solely FIDA measurements or combined FIDA and NES measurements. Here the density of ions with positive pitches exceeds the density of negative-pitch ions by less than $10 \%$ for $20 \mathrm{keV}<E<60 \mathrm{keV}$. On the other hand, the reconstructions in the slowing-down basis both suggest a density fraction of $n^{-} / n^{+}=1.3$, i.e. a smaller than expected but detectable density asymmetry about $p=0$. As for discharge \#75448, the disagreement between reconstructions and TRANSP in current density produced by ions with $E>20 \mathrm{keV}$ is likely to be explained by the relatively large signal in the blue-shifted range of the B-port FIDA measurements along with the complex shape of the dual-beam distribution.

\section{Conclusions and outlook}

Measurements from the two-view FIDA diagnostic setup installed at EAST enables reconstructions of NBI-generated fast-ion velocity distributions for energies above $\sim 15 \mathrm{keV}$ in the plasma center. With the incomplete coverage of velocity space and the limited amount of data provided by a two-view FIDA system, prior information must be imposed on reconstructions. Using a standard non-negativity constrained zeroth-order Tikhonov regularization on pixels provides solutions that overall distributes the fast ions accurately and allows identification of high- and low-density regions when employing nullmeasurements. The zeroth-order Tikhonov pixelreconstructions, however, tend to lose resolution of the fast-ion birth velocities and are strongly dependent on including null-measurements as prior information. The reconstructions are significantly improved by introducing a new approach to the toolbox of fastion velocity-space tomography: namely the utilization of the neoclassical slowing down of fast ions through Coulomb collisions in a fusion plasma. This is achieved by expressing the distribution as a linear combination of a selection of slowing-down distribution functions and perform the reconstruction in this basis. For FIDA-based reconstructions at EAST, this approach is beneficial in order to obtain smooth solutions with reduced artefacts and improved velocity-space resolution. Restricting the slowing-down basis to contain only a limited number of judiciously-chosen basis functions, based on e.g. geometric consideration of neutral beam injection, is likely to, furthermore, enable velocity-space reconstructions from single-view measurements, which has until now been unattainable. Examples of systems that are likely to benefit from the restricted basis function approach are FIDA measurements at MAST [14, TCV [51] and NSTX 52.53, CTS measurements at Wendelstein 7-X 54 and LHD [55], as well as NES and GRS measurements at JET $56,57$.

The expansion in base functions will, additionally, be essential for fast-ion phase-space inversion problems with sparse fast-ion measurements. Using full phasespace distribution functions computed by numerical simulations as base functions, it should be feasible to infer the distribution in the entire phase-space rather than in a single point as in velocity-space tomography. This method could be used in tokamaks in cases where an insufficient amount of measured data is available to do orbit tomography 58, 59]. Furthermore, the method is applicable to stellarators where the phasespace distribution is 5D (3D in position space and $2 \mathrm{D}$ in velocity space) which cannot be covered by measurements at present.

Derived quantities, computed as moments of the distribution, are consistent using both the pixel basis and the slowing-down basis in reconstructions with null-measurements. For purely co-current neutral beam injection at EAST, the fast-ion density, pressure and current density derived from measurement-based reconstructions are well-modelled by the neoclassical TRANSP distribution. For simultaneous co- and counter-current injection, on the other hand, no consistently good agreement between the modelled and reconstructed quantities is achieved. This is likely explained by three factors: uncertain absolute calibration of measurements, the relatively complex 
structure of fast-ion distributions generated from simultaneous co- and counter-current injection, or the disagreement between measurements and simulation at small blue-shifts in the B-port FIDA signals that might be caused by passive FIDA emission.

The good spatial localization as well as temporal and velocity-space resolution offered by the FIDA diagnostics along with the framework presented in this work will allow investigation of the interactions between fast ions and collective modes in future EAST campaigns and is an encouraging approach to study potential bulk heating through parametric decay of fast-ion-driven toroidal Alfvén eigenmodes proposed in 60.

Combining FIDA measurements with fast-ion sensitive NES measurements increases the available diagnostic information in the tomographic problem. The framework for this is illustrated in reconstructions of a fast-ion distribution generated from simultaneous co- and counter-current neutral beam injection. Here, we employed combined FIDA and NES measurements, obtained from the single-plate EJ301 scintillator. For the considered discharge, the improved high-energy velocity-space sensitivity offered by the FIDA+NES setup compared to the FIDA-only setup ultimately obviated null-measurements in the standard nonnegative zeroth-order Tikhonov pixel reconstruction. This effect was, however, less pronounced for slowingdown reconstructions.

At higher energies than considered here, the sensitivity of NES diagnostics strongly surpasses the FIDA sensitivity due to the strong relativeenergy dependence of both the fusion reaction crosssection and the charge-exchange probabilities. Hence, combining FIDA with NES provides a possibility to simultaneously reconstruct a large energy range (keV to $\mathrm{MeV}$ ), somewhat similar to the CTS/GRS symbiosis planned for ITER [16]. Additionally, extending the diagnostic suite by the time-of-flight neutron spectrometer TOFED and the single-plate stilbene scintillator 32 35, 37 will further improve the highenergy range of fast-ion velocity-space reconstructions, enabling detailed studies on the full fast-ion population during combined NBI and ICRF heating.

\section{Acknowledgments}

We appreciate the support of the ITPA Topical Group for Energetic Particle Physics and the EAST team. This work has been carried out within the framework of the EUROfusion Consortium and has received funding from the Euratom research and training programme 2014-2018 and 2019-2020 under grant agreement No. 633053. This work is, furthermore, supported by the National Natural Science Foundation of China under
Grant No. 11975276 and Anhui Provincial Natural Science Foundation No. 2008085J04. The views and opinions expressed herein do not necessarily reflect those of the European Commission.

\section{References}

[1] A Fasoli et al. Chapter 5: Physics of energetic ions. Nucl. Fusion, 47(6):S264-S284, 2007.

[2] R. Ding et al. Material migration studies with an ITER first wall panel proxy on EAST. Nucl. Fusion, 55(2):023013, 2015.

[3] ITER Physics Expert Group on Energetic Particles, Heating and Current Drive and ITER Physics Basis Editors. Chapter 5: Physics of energetic ions. Nucl. Fusion, 39:2471, 1999.

[4] S. D. Pinches et al. The role of energetic particles in fusion plasmas. Plasma Phys. Control. Fusion, 46:B187-B200, 2004.

[5] Y. Wan et al. Overview of the present progress and activities on the CFETR. Nucl. Fusion, 57(10):102009, 2017.

[6] G. Zhuang et al. Progress of the CFETR design. Nucl. Fusion, 59(11):112010, 2019.

[7] M. Salewski et al. Tomography of fast-ion velocityspace distributions from synthetic CTS and FIDA measurements. Nucl. Fusion, 52:103008, 2012.

[8] M. Salewski et al. Combination of fast-ion diagnostics in velocity-space tomographies. Nucl. Fusion, 53:063019, 2013.

[9] M. Salewski et al. Measurement of a 2D fast-ion velocity distribution function by tomographic inversion of fast-ion D-alpha spectra. Nucl. Fusion, 54(2):023005, 2014.

[10] M. Salewski et al. Deuterium temperature, drift velocity, and density measurements in non-maxwellian plasmas at ASDEX upgrade. Nucl. Fusion, 58:036017, 2018.

[11] M. Weiland et al. Enhancement of the FIDA diagnostic at ASDEX upgrade for velocity space tomography. Plasma Phys. Control. Fusion, 58:025012, 2016.

[12] M. Weiland et al. Phase-space resolved measurement of 2nd harmonic ion cyclotron heating using FIDA tomography at the ASDEX upgrade tokamak. Nucl. Fusion, 57:116058, 2017.

[13] A. S. Jacobsen et al. Inversion methods for fast-ion velocity-space tomography in fusion plasmas. Plasma Phys. Control. Fusion, 58:045016, 2016.

[14] B. Madsen et al. Velocity-space tomography using prior information at MAST. Rev. Sci. Instrum., 89:10D125, 2018.

[15] B. Madsen et al. Tomography of the positive-pitch fast-ion velocity distribution in DIII-D plasmas with Alfvén eigenmodes and neoclassical tearing modes. Nucl. Fusion, 60(6):066024, 2020.

[16] M. Salewski et al. Alpha-particle velocity-space diagnostic in ITER. Nucl. Fusion, 58:096019, 2018.

[17] Y. M. Hou et al. Fast-ion $\mathrm{D} \alpha$ spectrum diagnostic in the EAST. Rev. Sci. Instrum., 87:11E552, 2016.

[18] J. Zhang et al. Fast ion D-alpha measurements using a bandpass-filtered system on EAST. Rev. Sci. Instrum., 89:10D121, 2018.

[19] B. Madsen et al. Velocity-space tomography from synthetic FIDA measurements at EAST. In 45th EPS Conference on Plasma Physics, EPS 2018, pages 501-504. European Physical Society, 2018.

[20] M. Salewski et al. High-definition velocity-space tomography of fast-ion dynamics. Nucl. Fusion, 56:106024, 2016.

[21] M. Salewski et al. On velocity space interrogation regions 
of fast-ion collective thomson scattering at ITER. Nucl. Fusion, 51(8):083014, 2011.

[22] W. W. Heidbrink et al. Measurements of fast-ion acceleration at cyclotron harmonics using Balmer-alpha spectroscopy. Plasma Phys. Control. Fusion, 49:1457$1475,2007$.

[23] M. Salewski et al. On velocity-space sensitivity of fast-ion D-alpha spectroscopy. Plasma Phys. Control. Fusion, 56:105005, 2014.

[24] J. Galdon-Quiroga et al. Velocity-space sensitivity and tomography of scintillator-based fast-ion loss detectors. Plasma Phys. Control. Fusion, 60(10):105005, 2018.

[25] A. S. Jacobsen et al. Velocity-space sensitivity of neutron spectrometry measurements. Nucl. Fusion, 55(5):053013, 2015.

[26] A. S. Jacobsen et al. Velocity-space sensitivities of neutron emission spectrometers at the tokamaks JET and ASDEX Upgrade in deuterium plasmas. Rev. Sci. Instrum., 88(7):073506, 2017.

[27] M. Salewski et al. Velocity-space observation regions of high-resolution two-step reaction gamma-ray spectroscopy. Nucl. Fusion, 55(9):093029, 2015.

[28] M. Salewski et al. Fast-ion energy resolution by onestep reaction gamma-ray spectrometry. Nucl. Fusion, 56(4):046009, 2016.

[29] A. S. Jacobsen et al. Benchmark and combined velocityspace tomography of fast-ion D-alpha spectroscopy and collective Thomson scattering measurements. Plasma Phys. Control. Fusion, 58:042002, 2016.

[30] M. Salewski et al. MeV-range velocity-space tomography from gamma-ray and neutron emission spectrometry measurements at JET. Nucl. Fusion, 57:056001, 2017.

[31] M. Salewski et al. Bayesian integrated data analysis of fastion measurements by velocity-space tomography. Fusion Sci. Tech., 74:23-36, 2018.

[32] L. J. Ge et al. Velocity-space sensitivity of time-of-flight neutron spectrometer at EAST in deuterium plasma. Rev. Sci. Instrum., 89:10I143, 2018.

[33] L. J. Ge et al. Neutron emission spectroscopy measurements with a compact liquid scintillation detector for NBI-heated plasma at EAST. Plasma Phys. and Control. Fusion, 60:095004, 2018.

[34] Y. M. Zhang et al. Velocity-space sensitivity of the compact neutron emission spectrometers at EAST. Rev. Sci. Instrum., 89:10I141, 2018.

[35] X. Y. Peng et al. Measurement and simulation of the response function of time of flight enhanced diagnostics neutron spectrometer for beam ion studies at EAST tokamak. Rev. Sci. Instrum., 87:11D836, 2016.

[36] W. W. Heidbrink et al. Fast-ion D $\alpha$ measurements of the fast-ion distribution (invited). Rev. Sci. Instrum., 81:10D727, 2010.

[37] X. Zhang et al. Diagnosing NB plasmas on the EAST tokamak with new time-of-flight neutron spectrometer. Nucl. Fusion, 54(10):104008, 2014.

[38] A. Pankin et al. The tokamak Monto Carlo fast ion module NUBEAM in the National Transport Code Collaboration library. Comput. Phys. Commun., 159:157, 2004.

[39] D. Moseev and M. Salewski. Bi-maxwellian, slowing-down, and ring velocity distributions of fast ions in magnetized plasmas. Phys. Plasmas, 26(2):020901, 2019.

[40] W. W. Heidbrink et al. Hydrogenic fast-ion diagnostic using Balmer-alpha light. Plasma Phys. Control. Fusion, 46:1855-1875, 2004.

[41] D. Moseev et al. Recent progress in fast-ion diagnostics for magnetically confined plasmas. Rev. Mod. Plasma Phys., 2:7, 2018.

[42] J. Huang et al. Validation of fast-ion D-alpha spectrum measurements during EAST neutral-beam heated plasmas. Rev, Sci. Instrum., 87:11E542, 2016.
[43] B. Geiger et al. Progress in modelling fast-ion Dalpha spectra and neutral particle analyzer fluxes using FIDASIM. Plasma Phys. Control. Fusion, 62(10):105008, 2020.

[44] L. Stagner, B. Geiger, and W. W. Heidbrink. FIDASIM: A Neutral Beam and Fast-ion Diagnostic Modeling Suite. https://doi.org/10.5281/Zenodo.1341369

[45] W. W. Heidbrink et al. A code that simulates fast-ion $\mathrm{D}_{\alpha}$ and neutral particle measurements. Commun. Comput. Phys., 10:716741, 2011.

[46] X. Yuan et al. Neutron energy spectrum measurements with a compact liquid scintillation detector on EAST. JINST, 8:P07016, 2013

[47] R. C. Aster et al. Chapter five - discretizing inverse problems using basis functions. In Parameter Estimation and Inverse Problems (Third Edition), pages 135 - 149. Elsevier, third edition edition, 2019.

[48] M. G. von Hellermann et al. Observation of alpha particle slowing-down spectra in JET helium beam fuelling and heating experiments Plasma Phys. Control. Fusion, 35(7):799-824, 1993.

[49] W. G. F. Core. A note on the calculation of NBI fast ion distribution functions. Nucl. Fusion, 33(5):829-830, 1993.

[50] P. C. Hansen. Oblique projections and standardform transformations for discrete inverse problems. Numer. Linear Algebra Appl., 20(2):250-258, 2013.

[51] B. Geiger et al. Fast-ion transport in low density Lmode plasmas at TCV using FIDA spectroscopy and the TRANSP code. Plasma Phys. Control. Fusion, 59(11):115002, 2017.

[52] W. W. Heidbrink et al. Analysis of fast-ionD $\alpha$ data from the National Spherical Torus Experiment. Nucl. Fusion, 56(5):056005, 2016.

[53] D. Liu et al. Effect of sawtooth crashes on fast ion distribution in NSTX-U. Nucl. Fusion, 58(8):082028, 2018.

[54] I. Abramovic et al. Collective Thomson scattering data analysis for Wendelstein 7-X. J. Inst., 12(08):C08015C08015, 2017.

[55] M. Nishiura et al. Spectrum response and analysis of $77 \mathrm{GHz}$ band collective Thomson scattering diagnostic for bulk and fast ions in LHD plasmas. Nucl. Fusion, 54(2):023006, 2014.

[56] J. Eriksson et al. Measuring fast ions in fusion plasmas with neutron diagnostics at JET. Plasma Phys. Control. Fusion, 61(1):014027, 2018.

[57] M. Nocente et al. MeV range particle physics studies in tokamak plasmas using gamma-ray spectroscopy. Plasma Phys. Control. Fusion, 62(1):014015, 2019.

[58] L. E. Stagner. Inference of the fast-ion distribution function. PhD thesis, University of California Irvine, 2018.

[59] L. Stagner et al. Orbit tomography of energetic particle distribution functions. (submitted).

[60] Z. Qiu et al. Nonlinear decay and plasma heating by a toroidal Alfvén eigenmode. Phys. Rev. Lett., 120:135001, 2018. 